\title{
Loop Diuretic Use at Discharge Is Associated With Adverse Outcomes in Hospitalized Patients With Heart Failure
}

\author{
- A Report From the Japanese Cardiac Registry of \\ Heart Failure in Cardiology (JCARE-CARD) -
}

\author{
Sanae Hamaguchi, MD, PhD; Shintaro Kinugawa, MD, PhD; Miyuki Tsuchihashi-Makaya, PhD; \\ Daisuke Goto, MD, PhD; Satoshi Yamada, MD, PhD; Hisashi Yokoshiki, MD, PhD; \\ Akira Takeshita, MD, PhD; Hiroyuki Tsutsui, MD, PhD; The JCARE-CARD Investigators
}

\begin{abstract}
Background: Loop diuretics are commonly used in patients with heart failure (HF) to remove retained fluid and improve symptoms. However, they may potentially worsen outcomes in HF. It remains unknown whether the use of loop diuretics is associated with adverse HF outcomes in routine clinical practice. We thus determined the effects of loop diuretic use at discharge on long-term mortality and rehospitalization among patients hospitalized with HF.
\end{abstract}

Methods and Results: The Japanese Cardiac Registry of Heart Failure in Cardiology (JCARE-CARD) prospectively studied the characteristics and treatments of a broad sample of patients hospitalized with worsening HF and followed for 2.1 years. Among a total of 2,549 HF patients, loop diuretics were used by 2,015 patients (79\%), but not 534 patients $(21 \%)$. The mean age was 70.7 years and $60 \%$ were male. Etiology was ischemic in $32 \%$ and mean left ventricular ejection fraction was $42 \%$. After adjustment for covariates, discharge use of loop diuretics was associated with significant adverse risks of cardiac death (adjusted hazard ratio [HR] 2.348, 95\% confidence interval [Cl] 1.246-4.423, P=0.008) and rehospitalization (adjusted HR 1.427, 95\% Cl 1.040-1.959, $\mathrm{P}=0.027$ ).

Conclusions: Among patients hospitalized with worsening HF, loop diuretic use at discharge was associated with long-term adverse outcomes, which suggests that routine chronic use of loop diuretics may be harmful for patients with HF. (Circ J 2012; 76: 1920-1927)

Key Words: Diuretics; Heart failure; Outcomes; Prognosis

$\mathbf{L}$ oop diuretics are the only drugs that can effectively control fluid retention in patients with heart failure (HF) and fluid overload..$^{1,2}$ However, loop diuretics can reduce the glomerular filtration rate (GFR), further worsen neurohormonal activation, and cause electrolyte disturbances. ${ }^{3,4}$ Furthermore, they increase myocardial fibrosis, $, 5,6$ which may be associated with disease progression and poor prognosis of HF. There have been no randomized clinical trials to determine the chronic effects of loop diuretics on HF outcomes. Previous subanalyses of randomized clinical trials demonstrated that the use of diuretics was associated with adverse outcomes in patients with $\mathrm{HF}$ and reduced left ventricular ejection fraction (LVEF). $4,7,8$ In the Studies Of Left Ventricular Dysfunction (SOLVD), baseline use of a non-potassium-sparing diuretic was associated with an increased risk of arrhythmic death, after controlling for other variables of disease se- verity. ${ }^{4}$ Similarly, analysis of the data from the Evaluation Study of Congestive Heart Failure and Pulmonary Artery Catheter Effectiveness (ESCAPE) trial demonstrated that higher doses of diuretics were associated with increased mortality over 6 months of follow-up among patients hospitalized with advanced HF. ${ }^{7}$ However, these analyses used the data for HF patients enrolled in randomized clinical trials, clearly different from those in the "real world" under current standard practice for $\mathrm{HF}$ treatment, who are more elderly and have more comorbidities. In fact, in those trials, patients with HF and preserved LVEF or renal dysfunction were excluded. Thus, the effect of loop diuretics on long-term outcomes needed to be assessed in an unselected population of HF patients.

The Japanese Cardiac Registry of Heart Failure in Cardiology (JCARE-CARD) prospectively studied the characteristics, treatments and outcomes, including death and rehospitaliza-

Received October 17, 2011; revised manuscript received April 9, 2012; accepted April 10, 2012; released online May 26, 2012 Time for primary review: 20 days

Department of Cardiovascular Medicine, Hokkaido University Graduate School of Medicine, Sapporo, Japan

Dr Akira Takeshita died in March 2009.

Mailing address: Hiroyuki Tsutsui, MD, PhD, Department of Cardiovascular Medicine, Hokkaido University Graduate School of Medicine,

Kita-15, Nishi-7, Kita-ku, Sapporo 060-8638, Japan. E-mail: htsutsui@med.hokudai.ac.jp

ISSN-1346-9843 doi:10.1253/circj.CJ-11-1196

All rights are reserved to the Japanese Circulation Society. For permissions, please e-mail: cj@j-circ.or.jp 


\begin{tabular}{|c|c|c|c|c|}
\hline Characteristics & $\begin{array}{c}\text { Total } \\
(\mathrm{n}=2,549)\end{array}$ & $\begin{array}{l}\text { Loop diuretic } \\
\text { use }(n=2,015)\end{array}$ & $\begin{array}{l}\text { No loop diuretic } \\
\text { use }(n=534)\end{array}$ & $P$ value \\
\hline Age, years (mean $\pm S D$ ) & $70.7 \pm 13.3$ & $70.9 \pm 13.2$ & $69.8 \pm 13.7$ & 0.090 \\
\hline Male, \% & 60.0 & 60.9 & 56.6 & 0.066 \\
\hline BMI, $\mathbf{k g} / \mathrm{m}^{2}$ & $22.4 \pm 4.1$ & $22.4 \pm 4.1$ & $22.2 \pm 4.1$ & 0.262 \\
\hline \multicolumn{5}{|l|}{ Causes of HF, \% } \\
\hline Ischemic & 32.0 & 33.1 & 28.1 & 0.029 \\
\hline Valvular & 27.7 & 28.8 & 23.6 & 0.016 \\
\hline Hypertensive & 24.2 & 23.5 & 26.8 & 0.118 \\
\hline Dilated cardiomyopathy & 18.4 & 18.4 & 18.4 & 0.975 \\
\hline \multicolumn{5}{|l|}{ Medical history, \% } \\
\hline Hypertension & 52.8 & 52.7 & 53.4 & 0.778 \\
\hline Diabetes mellitus & 30.0 & 31.4 & 24.6 & 0.002 \\
\hline Dyslipidemia & 25.1 & 26.1 & 21.4 & 0.028 \\
\hline Hyperuricemia & 46.2 & 48.3 & 37.9 & $<0.001$ \\
\hline Prior stroke & 14.4 & 14.9 & 12.7 & 0.211 \\
\hline COPD & 6.5 & 6.7 & 5.9 & 0.545 \\
\hline Smoking & 38.1 & 38.8 & 35.6 & 0.191 \\
\hline Prior myocardial infarction & 27.0 & 28.7 & 20.9 & $<0.001$ \\
\hline Atrial fibrillation & 35.2 & 36.8 & 29.1 & 0.001 \\
\hline Sustained VT/VF & 6.0 & 6.3 & 4.7 & 0.163 \\
\hline \multicolumn{5}{|l|}{ Procedures, \% } \\
\hline $\mathrm{PCl}$ & 17.8 & 18.4 & 15.2 & 0.085 \\
\hline CABG & 9.5 & 10.2 & 6.8 & 0.020 \\
\hline Valvular surgery & 6.7 & 7.2 & 4.9 & 0.061 \\
\hline PPM & 0.9 & 1.0 & 0.6 & 0.349 \\
\hline ICD & 2.0 & 1.9 & 2.6 & 0.285 \\
\hline CRT & 1.6 & 1.6 & 1.5 & 0.820 \\
\hline \multicolumn{5}{|l|}{ Vital signs at discharge } \\
\hline NYHA functional class, $\%$ & & & & 0.043 \\
\hline 1 & 36.5 & 35.3 & 41.2 & \\
\hline 2 & 57.2 & 58.0 & 53.9 & \\
\hline 3 & 6.2 & 6.5 & 4.9 & \\
\hline 4 & 0.2 & 0.2 & 0 & \\
\hline Heart rate, beats/min & $70.3 \pm 11.8$ & $70.2 \pm 11.6$ & $70.7 \pm 12.6$ & 0.652 \\
\hline $\mathrm{SBP}, \mathrm{mmHg}$ & $117.3 \pm 18.3$ & $116.7 \pm 18.1$ & $119.2 \pm 19.2$ & 0.017 \\
\hline $\mathrm{DBP}, \mathrm{mmHg}$ & $66.2 \pm 10.4$ & $66.0 \pm 11.6$ & $66.9 \pm 11.3$ & 0.138 \\
\hline \multicolumn{5}{|l|}{ Laboratory data at discharge } \\
\hline eGFR, $\mathrm{ml} \cdot \mathrm{min}^{-1} \cdot 1.73 \mathrm{~m}^{-2}$ & $51.5 \pm 24.7$ & $50.8 \pm 24.1$ & $54.2 \pm 26.8$ & 0.005 \\
\hline Serum uric acid, mg/dl & $7.4 \pm 2.9$ & $7.4 \pm 2.3$ & $7.0 \pm 4.5$ & $<0.001$ \\
\hline Hemoglobin, g/dl & $12.1 \pm 3.3$ & $12.1 \pm 3.3$ & $12.3 \pm 2.9$ & 0.240 \\
\hline Plasma BNP, pg/ml & $375 \pm 474$ & $377 \pm 411$ & $369 \pm 658$ & 0.010 \\
\hline \multicolumn{5}{|l|}{ Echocardiographic data } \\
\hline LVEDD, mm & $56.3 \pm 10.4$ & $56.8 \pm 10.4$ & $54.6 \pm 10.3$ & $<0.001$ \\
\hline LVESD, mm & $53.0 \pm 9.4$ & $54.0 \pm 9.4$ & $52.2 \pm 9.3$ & $<0.001$ \\
\hline LVEF, \% & $42.2 \pm 17.5$ & $41.8 \pm 17.6$ & $43.3 \pm 17.1$ & 0.059 \\
\hline
\end{tabular}

Data are percent or means \pm SD.

$\mathrm{BMI}$, body mass index; HF, heart failure; COPD, chronic obstructive pulmonary disease; VT/VF, ventricular tachycardia/fibrillation; $\mathrm{PCl}$, percutaneous coronary intervention; CABG, coronary artery bypass grafting; ICD, implantable cardioverter defibrillator; CRT, cardiac resynchronization therapy; NYHA, New York Heart Association; SBP, systolic blood pressure; DBP, diastolic blood pressure; eGFR, estimated glomerular filtration rate; BNP, B-type natriuretic peptide; LVEDD, left ventricular end-diastolic diameter; LVESD, left ventricular end-systolic diameter; LVEF, left ventricular ejection fraction.

tion, in a broad sample of patients hospitalized with HF in Japan. ${ }^{9-20}$ The JCARE-CARD enrolled 2,675 patients admitted with HF and an average of follow-up of 2.2 years at 164 participating hospitals in a web-based registry.

The aim of the present study was to analyze the prognostic value of loop diuretics on mortality and rehospitalization rates by evaluating the relationship between the drugs' use at discharge and clinical outcomes among patients hospitalized with $\mathrm{HF}$ and registered in the JCARE-CARD database. 


\begin{tabular}{|c|c|c|c|c|}
\hline & $\begin{array}{c}\text { Total } \\
(\mathrm{n}=2,549)\end{array}$ & $\begin{array}{l}\text { Loop diuretic } \\
\text { use }(n=2,015)\end{array}$ & $\begin{array}{l}\text { No loop diuretic } \\
\text { use }(n=534)\end{array}$ & $P$ value \\
\hline ACEI, \% & 37.4 & 38.6 & 32.8 & 0.013 \\
\hline ARB, $\%$ & 44.4 & 43.7 & 47.2 & 0.146 \\
\hline ACEI or ARB, $\%$ & 76.5 & 76.8 & 75.5 & 0.511 \\
\hline ACEI and ARB, \% & 5.3 & 5.5 & 4.5 & 0.374 \\
\hline$\beta$-blocker, \% & 48.6 & 47.8 & 51.9 & 0.093 \\
\hline ACEI or ARB and $\beta$-blocker, $\%$ & 39.9 & 39.8 & 40.6 & 0.710 \\
\hline Thiazide, \% & 3.6 & 2.9 & 6.4 & $<0.001$ \\
\hline Spironolactone, \% & 41.6 & 47.6 & 18.7 & $<0.001$ \\
\hline Digitalis, \% & 30.9 & 31.7 & 27.7 & 0.075 \\
\hline Calcium-channel blocker, \% & 25.2 & 24.2 & 28.8 & 0.029 \\
\hline Nitrates, \% & 23.3 & 24.4 & 19.1 & 0.010 \\
\hline Antiarrhythmics, \% & 16.6 & 16.9 & 15.5 & 0.463 \\
\hline Aspirin, \% & 47.2 & 47.6 & 45.5 & 0.390 \\
\hline Warfarin, \% & 40.8 & 41.5 & 37.8 & 0.121 \\
\hline Statin, \% & 19.9 & 20.4 & 17.6 & 0.143 \\
\hline
\end{tabular}

$\mathrm{ACEl}$, angiotensin-converting enzyme inhibitor; ARB, angiotensin-receptor blocker.

\section{Methods}

\section{Patients}

The details of the JCARE-CARD have been described previously.9,10,14,15,19 Briefly, eligible patients were those hospitalized with worsening HF as the primary cause of admission. The study hospitals were encouraged to register the patients as consecutively as possible. For each patient, baseline data included (1) age, sex, and body mass index; (2) cause of HF; (3) medical history; (4) prior procedures; (5) vital signs at discharge; (6) laboratory data; (7) echocardiographic data; and (8) medication use at discharge. The data were entered using a web-based electronic data capture (EDC) system licensed by the JCARE-CARD (www.jcare-card.jp).

From the database of a total cohort of 2,675 patients registered in JCARE-CARD, the present analysis used the data of 2,549 patients for whom information of the loop diuretic use could be obtained. The patients were divided into 2 groups according to loop diuretic use $(n=2,015 ; 79.1 \%)$ or no loop diuretic use $(n=534 ; 20.9 \%)$ at the time of discharge from the index hospitalization.

\section{Outcomes}

The status of all patients was surveyed and the following information about outcomes was obtained from participating cardiologists using the web-based EDC system: (1) all-cause death, (2) cardiac death, defined as death due to HF, myocardial infarction or other causes such as pulmonary embolism, (3) rehospitalization due to an exacerbation of HF that required more than continuation of the patient's usual therapy on prior admission, and (4) a composite endpoint of all-cause death and rehospitalization due to HF. The endpoints were adjudicated by the cardiologists in each participating hospital. Of the 2,549 patients, 244 (9.6\%), missed during follow-up, were excluded from the follow-up analysis. Follow-up data could be obtained for 2,305 of the 2,549 patients $(90.4 \%)$. Of these 2,305 patients, 1,814 were in the group of loop diuretic use and 491 were in that of no loop diuretic use. Mean postdischarge follow-up was $781 \pm 315$ days $(2.1 \pm 0.9$ years $)$.

The hypothesis being tested was whether loop diuretic use at hospital discharge would be associated with higher mortal- ity and rehospitalization rates during the follow-up compared with no loop diuretic use.

\section{Statistical Analysis}

Patients' characteristics and treatments were compared using $\chi^{2}$ test for categorical variables, Student's t-test for normally distributed continuous variables, and Mann-Whitney U test for continuous variables not normally distributed. We analyzed the data excluding the patients with missing data. Only patients who survived the index hospitalization were included in the follow-up analysis. Cumulative event-free rates during the follow-up were derived using the method of Kaplan and Meier. The relationship between loop diuretic use at discharge and outcome was evaluated among patients with multivariable adjustment. The covariates of age, sex, estimated GFR (eGFR) at discharge, systolic blood pressure (SBP) at discharge, LVEF, B-type natriuretic peptide (BNP), New York Heart Association (NYHA) functional class at discharge, cause of HF (ischemic, valvular), medical history (diabetes, dyslipidemia, hyperuricemia, prior myocardial infarction, and atrial fibrillation) and medication use (angiotensin-converting enzyme inhibitor [ACEI], $\beta$-blocker, spironolactone, thiazide, calcium-channel blocker (CCB), nitrate, and statins), were used in developing the postdischarge Cox proportional hazard models. The same variables were included in a multivariable logistic regression model and the propensity score (PS) for loop diuretic use was estimated for each patient. Using a greedy matching protocol, we matched each patient with no loop diuretic use to a patient with loop diuretic use who had a very similar PS; thus we used 465 pairs matched with PS for PS matching. We performed a formal sensitivity analysis for unmeasured confounding factors. ${ }^{21}$

The results were reported as hazard ratio (HR), 95\% confidence interval (CI), and $\mathrm{P}$ value. HR for outcomes when loop diuretics were used was compared with no use of diuretics. A $P$ value of $<0.05$ was used as the criterion for variables to stay in the model. SPSS version $16.0 \mathrm{~J}$ for Windows was used for all statistical analyses (Chicago, IL, USA). 


\begin{tabular}{|c|c|c|c|c|c|}
\hline \multirow[b]{2}{*}{ Outcome } & \multicolumn{2}{|c|}{ n (\%) } & \multirow{3}{*}{ HR } & \multirow{3}{*}{$95 \% \mathrm{Cl}$} & \multirow{3}{*}{$P$ value } \\
\hline & $\begin{array}{l}\text { Loop diuretic } \\
\text { use }(n=1,814)\end{array}$ & $\begin{array}{l}\text { No loop diuretic } \\
\text { use }(n=491)\end{array}$ & & & \\
\hline All-cause death & $399(22.0 \%)$ & $75(15.3 \%)$ & & & \\
\hline Unadjusted & & & 1.501 & $1.171-1.925$ & 0.001 \\
\hline Adjusted for covariates & & & 1.545 & $0.986-2.420$ & 0.058 \\
\hline $\begin{array}{l}\text { Adjusted for matching with propensity } \\
\text { score }\end{array}$ & & & 1.510 & $1.113-2.048$ & 0.008 \\
\hline Cardiac death & $251(13.8 \%)$ & $41(8.4 \%)$ & & & \\
\hline Unadjusted & & & 1.703 & $1.224-2.370$ & 0.001 \\
\hline Adjusted for covariates & & & 2.348 & $1.246-4.423$ & 0.008 \\
\hline $\begin{array}{l}\text { Adjusted for matching with propensity } \\
\text { score }\end{array}$ & & & 1.719 & $1.155-2.560$ & 0.008 \\
\hline Rehospitalization & $690(38.0 \%)$ & $146(29.7 \%)$ & & & \\
\hline Unadjusted & & & 1.360 & $1.137-1.627$ & 0.001 \\
\hline Adjusted for covariates & & & 1.427 & $1.040-1.959$ & 0.027 \\
\hline $\begin{array}{l}\text { Adjusted for matching with propensity } \\
\text { score }\end{array}$ & & & 1.194 & $0.953-1.495$ & 0.124 \\
\hline All-cause death or rehospitalization & $834(46.0 \%)$ & $182(37.1 \%)$ & & & \\
\hline Unadjusted & & & 1.326 & $1.129-1.558$ & 0.001 \\
\hline Adjusted for covariates & & & 1.363 & $1.022-1.816$ & 0.035 \\
\hline $\begin{array}{l}\text { Adjusted for matching with propensity } \\
\text { score }\end{array}$ & & & 1.236 & $1.010-1.511$ & 0.040 \\
\hline
\end{tabular}

A Cox regression model was used in the analysis adjusted for the following covariates: age, sex, eGFR at discharge, SBP at discharge, LVEF, BNP, NYHA functional class at discharge, cause of HF (ischemic, valvular), medical history (diabetes mellitus, dyslipidemia, hyperuricemia, prior myocardial infarction, and atrial fibrillation) and medication use (ACEl, $\beta$-blocker, spironolactone, thiazide, calcium-channel blocker, nitrate, statin). The same variables were used to determine the propensity score for loop diuretic use, and 465 pairs were matched. The no loop diuretic use group was the reference group.

$\mathrm{HR}$, hazard ratio; $\mathrm{Cl}$, confidence interval. Other abbreviations as in Tables 1,2.

\section{Results}

\section{Patients' Characteristics}

The present study included 2,549 patients with a mean age of $70.7 \pm 13.3$ years and $60.0 \%$ were men (Table 1 ). The causes of $\mathrm{HF}$ were ischemic heart disease in $32.0 \%$, valvular heart disease in $27.7 \%$, hypertensive heart disease in $24.2 \%$, and dilated cardiomyopathy in $18.4 \%$. The mean LVEF was $42.2 \pm 17.5 \%$.

The characteristics of patients prescribed loop diuretics at discharge and those not prescribed are compared in Table 1. Those discharged with loop diuretics had significantly more ischemic and valvular HF etiologies; more comorbidities, such as diabetes, dyslipidemia, hyperuricemia, prior myocardial infarction, and atrial fibrillation; significantly more cases of coronary artery bypass grafting; severe HF symptoms by NYHA functional class at discharge; and SBP at discharge was significantly lower. However, diastolic blood pressure did not differ between groups. eGFR was significantly lower, and serum uric acid and plasma BNP levels were higher in patients with loop diuretics. LV end-diastolic and end-systolic diameters were significantly greater in patients with loop diuretics and LVEF tended to be lower, although this did not reach statistical significance $(\mathrm{P}=0.059)$.

Use of medications other than loop diuretics was compared between groups (Table 2). The use of ACEIs was slightly, but significantly, higher in patients with loop diuretic use $(38.6 \%$ vs. $32.8 \%, \mathrm{P}=0.013$ ). However, the use of ACEI or angiotensin-receptor blocker (ARB) did not differ between groups ( $76.8 \%$ vs. $75.5 \%, \mathrm{P}=0.511$ ). Importantly, the combination of ACEI or ARB and $\beta$-blocker was similar between groups. However, spironolactone and nitrate were more often prescribed for the patients with loop diuretic use. On the other hand, thiazide diuretic and CCBs were more used by the patients with no loop diuretic use.

\section{Postdischarge Clinical Outcomes According to Loop Diuretic Use}

During follow-up of 2.1 years after hospital discharge, the rates of adverse outcomes were as follows: all-cause death $20.6 \%$, cardiac death $12.7 \%$, rehospitalization due to the worsening $\mathrm{HF}$ $36.3 \%$, and all-cause death or rehospitalization $44.1 \%$. The unadjusted rates of all-cause death, cardiac death, rehospitalization due to the worsening $\mathrm{HF}$, and all-cause death or rehospitalization were significantly higher in patients with loop diuretic use (Table 3, Figure).

Even after adjustment for covariates in the multivariable Cox proportional hazard models, discharge use of loop diuretics, when compared to no use, was associated with an increased risk of cardiac death (HR 2.348, 95\% CI 1.246-4.423, $\mathrm{P}=0.008$ ), rehospitalization (HR 1.427, 95\% CI 1.040-1.959, $\mathrm{P}=0.027$ ), and all-cause death or rehospitalization (HR 1.363, 95\% CI 1.022-1.816, $\mathrm{P}=0.035$ ) (Table 3). Patients taking loop diuretics tended to have a higher risk of all-cause death, which, however, did not reach statistical significance after adjustment $(\mathrm{P}=0.058)$.

Furthermore, when we matched by PS, the same variables shown in Table 1 and Table 2 were comparable between groups. Even after adjustment for matching with PS, loop diuretic use was associated with all-cause death (HR 1.510, 95\% CI 1.113-2.048, $\mathrm{P}=0.008)$, cardiac death (HR 1.719, 95\% CI 1.155-2.560, $\mathrm{P}=0.008)$, and all-cause death and rehospitalization (HR 1.236, 95\% CI 1.010-1.511, $\mathrm{P}=0.040)$. Sensitivity 

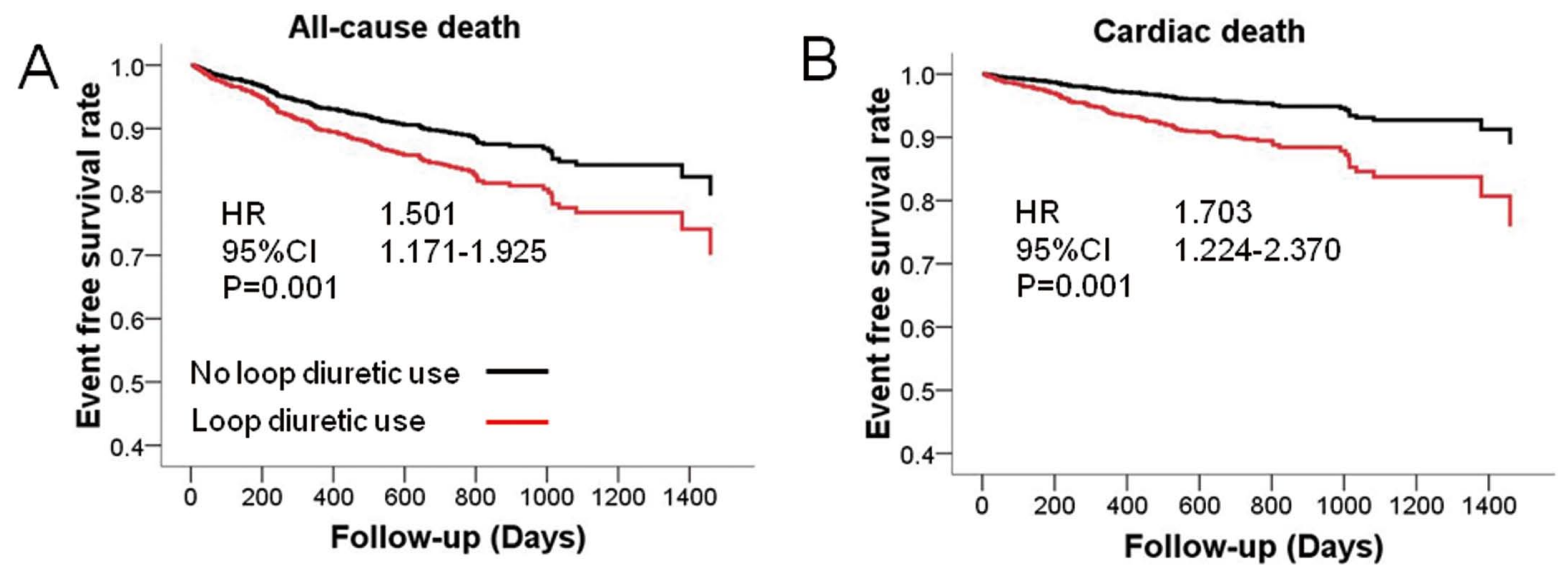

Rehospitalization
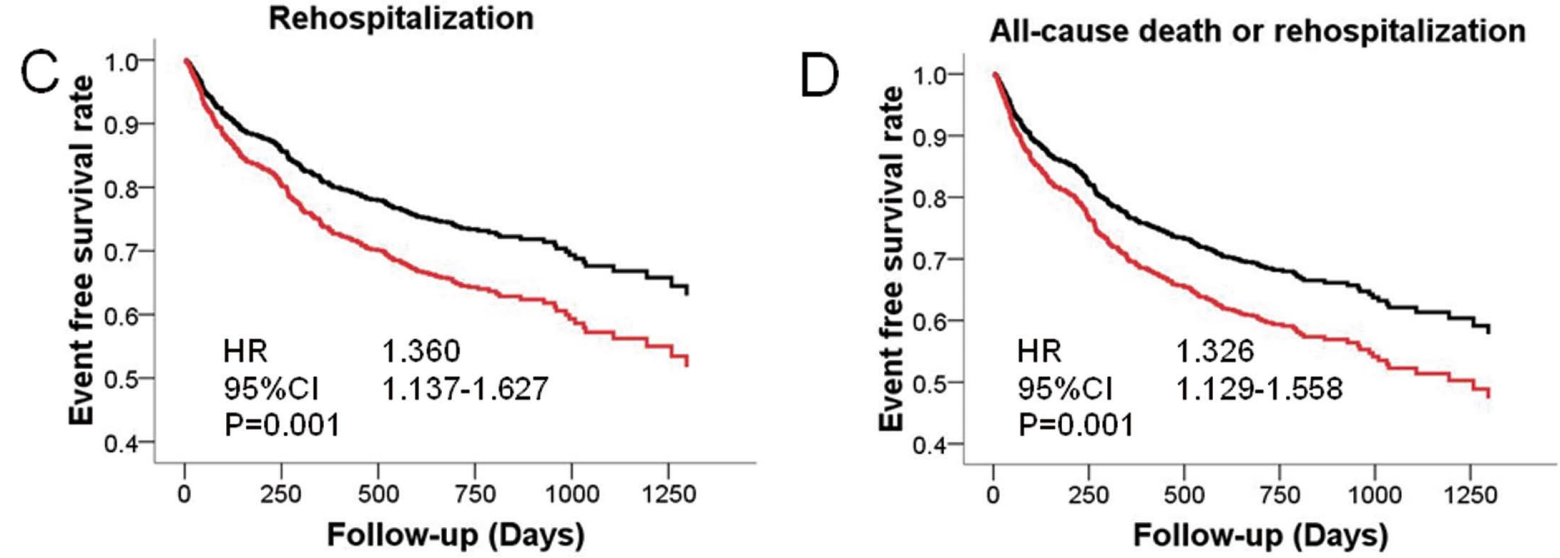

Figure. Kaplan-Meier survival curves for free from all-cause death (A), cardiac death (B), rehospitalization due to worsening heart failure (C), and all-cause death or rehospitalization (D) in hospitalized patients with loop diuretic use (red lines, $n=1,814$ ) vs. no loop diuretic use (black lines, $\mathrm{n}=491$ ) at discharge. HR, hazard ratio; $\mathrm{Cl}$, confidence interval.

analysis for unmeasured confounding factors was performed. In the absence of unmeasured confounding factors, a binomial test for matched pair provides strong evidence $(\mathrm{P}=0.003)$ that loop diuretic use increases cardiac death, even after adjustment by PS matching. To attribute the higher rate of cardiac death to unmeasured confounding factor rather than to an effect of loop diuretic use, that unmeasured confounding factor would need to produce a $27 \%$ increase in the odds of loop diuretic use, and it would need to be a strong predictor of cardiac death.

\section{Subgroup Analyses}

The association of loop diuretic use with cardiac death was noted across a wide spectrum of HF patients (Table 4). Loop diuretic use was associated with increased cardiac death in HF patients who were elderly ( $\geq 70$ years), with a non-ischemic etiology, no hypertension, no diabetes, and LVEF $\geq 40 \%$. In any subgroup, there were no significant interactions between groups.

\section{Discussion}

The present study using the JCARE-CARD database demonstrated that among patients hospitalized with worsening HF, loop diuretic use at discharge was associated with adverse out- comes during long-term follow-up up to 2.1 years.

These findings confirm and extend the results of previous studies that suggested an association between diuretic use and worse outcomes in patients with HF., 4,8,22 In the SOLVD trial, use of a diuretic was associated with a $37 \%$ increase in the risk of arrhythmic death after controlling for multiple other variables of disease severity. ${ }^{4}$ The Digitalis Investigation Group Study also found a $31 \%$ increased risk of death associated with diuretic use when using propensity matching to control for baseline differences in patients with and without diuretic use. ${ }^{8}$ The ESCAPE trial demonstrated a linear relationship between loop diuretic dose and mortality over 6 months of follow-up in patients hospitalized with HF. ${ }^{7}$ Moreover, in a cohort of 1,354 patients with advanced HF and reduced LVEF referred to a single center, there was a dose-dependent association between loop diuretic use and impaired survival during 2-year follow-up.22 However, those previous studies were performed with data from patients enrolled in clinical trials or cohort studies of patients with HF and reduced LVEF, thus excluding patients with HF and preserved LVEF. In contrast, the present study included patients hospitalized due to worsening HF as the primary cause of admission independent of LVEF data. Therefore, our findings confirm the association between loop diuretic use and poor outcomes in the "real world" under current standard medical practice. Furthermore, the present results 


\begin{tabular}{|lrcccc}
\hline \multicolumn{7}{l}{ Table 4. Subgroup Analyses of Cardiac Death According to Loop Diuretic Use } & & \\
\multicolumn{1}{c}{ Subgroup } & $\mathbf{n}$ & HR & $\mathbf{9 5 \%} \mathbf{C l}$ & $\mathbf{P}$ value & $\begin{array}{c}\text { P value } \\
\text { for interaction }\end{array}$ \\
Age $<70$ years & 936 & 1.812 & $0.927-3.542$ & 0.082 & \\
Age $\geq 70$ years & 1,369 & 1.599 & $1.094-2.338$ & 0.015 & 0.759 \\
Male & 1,376 & 1.604 & $1.042-2.471$ & 0.032 & \\
Female & 929 & 1.842 & $1.102-3.078$ & 0.020 & 0.679 \\
Ischemic & 727 & 1.670 & $0.934-2.985$ & 0.084 & \\
Non-ischemic & 1,578 & 1.691 & $1.131-2.528$ & 0.010 & 0.975 \\
Hypertension & 1,203 & 1.620 & $0.967-2.712$ & 0.067 & \\
No hypertension & 1,087 & 1.758 & $1.143-2.705$ & 0.010 & 0.803 \\
Diabetes & 675 & 1.370 & $0.726-2.584$ & 0.331 & \\
No diabetes & 1,626 & 1.826 & $1.239-2.690$ & 0.002 & 0.443 \\
LVEF $\geq 40 \%$ & 1,020 & 2.484 & $1.394-4.428$ & 0.002 & \\
LVEF $<40 \%$ & 1,000 & 1.207 & $0.761-1.913$ & 0.425 & 0.055 \\
\hline
\end{tabular}

No loop diuretic use group was a reference.

Abbreviations as in Tables 1,3.

were consistent with our own previous study, in which the use of diuretics was independently associated with higher mortality in elderly patients. ${ }^{9}$

There are several potential mechanisms by which loop diuretics may be associated with adverse outcomes in HF patients. First, administration of diuretics to patients with HF may activate the renin-angiotensin-aldosterone system, as well as the sympathetic nervous system, both of which play a detrimental role in HF progression. ${ }^{23-25}$ Neurohormonal activation is known to occur in patients with HF before overt symptoms appear. Plasma renin activity and norepinephrine levels have been shown to be significantly higher in HF patients with symptoms than in those without them. ${ }^{25}$ Moreover, plasma renin activity is normal in HF patients without symptoms and who are not using diuretics and is significantly increased in patients on diuretic therapy. ${ }^{25}$ In a tachycardia-induced animal model of HF, loop diuretic use significantly accelerated LV systolic dysfunction, elevated the serum aldosterone level, and increased basal sodium-calcium exchanger currents. ${ }^{6}$ Activation of the renin-angiotensin-aldosterone system induces myocardial fibrosis, oxidative stress, stimulation of proinflammatory cytokines, and myocardial fibrosis. ${ }^{26-29}$ Second, loop diuretics may also decrease intravascular volume and the GFR, which may also be caused by neurohormonal activation. Previous studies, including our own, have demonstrated that renal dysfunction is a common and independent risk for cardiovascular adverse outcomes in HF patients. ${ }^{11,30-39}$ Finally, loop diuretics cause electrolyte imbalances, such as decreases in potassium and magnesium, ${ }^{40,41}$ which may increase the risk of fatal arrhythmias and sudden cardiac death. ${ }^{40-44}$

\section{Clinical Implications}

Based on findings suggesting an association between diuretic use and worsening outcomes in patients with HF, guidelines from the European Society of Cardiology recommend that diuretics be used for HF patients with clinical symptoms or signs of volume overload and congestion. ${ }^{45}$ The practice guideline from the Heart Failure Society of America also recommends loop diuretics at doses needed to produce diuresis sufficient to achieve an optimal volume status. ${ }^{46}$ Therefore, routine chronic use of loop diuretics for HF patients without fluid retention needs to be avoided.

\section{Study Limitations}

Several limitations inherent in the design of the registry should be considered. First, the documentation of loop diuretic use at hospital discharge might not accurately reflect continuation over time or start after discharge. Moreover, we did not collect information regarding the dose and type of loop diuretic, such as furosemide and torsemide, and whether loop diuretics were initiated during or before hospitalization. Therefore, we could not assess the dose-effect relation in the study patients. However, higher doses are associated with higher mortality, based on the results of the ESCAPE trial. ${ }^{7}$ Second, information regarding the serum electrolyte concentration was not obtained in this database, so we could not assess the impact of hypokalemia, hyponatremia, and hypomagnesemia on outcomes. Third, the present study was not a prospective randomized trial and, despite covariate adjustment and adjustment for matching by PS and sensitivity analysis, other measured and unmeasured factors may have influenced the outcomes. Renal dysfunction, hyperuricemia and electrolyte imbalances commonly associated with loop diuretic use might affect the outcomes. Specifically, patients who received loop diuretics might do so because of greater disease severity compared to those who did not. However, the present study demonstrated an adverse effect of loop diuretics, even after extensive multivariable adjustment of other known predictors and adjustment for PS matching, and it was consistent among the different subgroups studied. Moreover, this has been persistently reported in most prior studies. ${ }^{4,7,8,22}$ Even though the present finding that use of loop diuretics was associated with worse outcomes is consistent with similar previous reports, unmeasured or unanalyzed factors that motivated the physicians caring for HF patients to give loop diuretics could have also put these patients at higher risk for death and subsequent hospitalization. It is difficult, even impossible, to account for these factors and the conclusion of the present study could be considered as "hypothesis generating". Finally, data were dependent on the accuracy of documentation and abstraction by the individual medical centers that participated in this study.

\section{Conclusions}

Among patients hospitalized with worsening HF, loop diuretic use at discharge was associated with adverse outcomes during a long-term follow-up of up to 2.1 years. The potential risk of 
loop diuretics for the larger numbers of HF patients encountered in routine clinical practice was suggested.

\section{Acknowledgments}

The JCARE-CARD investigators and participating cardiologists are listed in the Appendix of our previous publication. ${ }^{10}$ This study could not have been carried out without the help, cooperation and support of the cardiologists in the survey institutions. We thank them for allowing us to obtain the data. The JCARE-CARD was supported by the Japanese Circulation Society and the Japanese Society of Heart Failure and by grants from Health Sciences Research Grants from the Japanese Ministry of Health, Labor and Welfare (Comprehensive Research on Cardiovascular Diseases), the Japan Heart Foundation, and Japan Arteriosclerosis Prevention Fund.

\section{References}

1. Hunt SA. ACC/AHA 2005 guideline update for the diagnosis and management of chronic heart failure in the adult: A report of the American College of Cardiology/American Heart Association Task Force on Practice Guidelines (Writing Committee to Update the 2001 Guidelines for the Evaluation and Management of Heart Failure). J Am Coll Cardiol 2005; 46: e1-e82.

2. Jessup M, Abraham WT, Casey DE, Feldman AM, Francis GS, Ganiats TG, et al. 2009 focused update: ACCF/AHA Guidelines for the Diagnosis and Management of Heart Failure in Adults: A report of the American College of Cardiology Foundation/American Heart Association Task Force on Practice Guidelines: Developed in collaboration with the International Society for Heart and Lung Transplantation. Circulation 2009; 119: 1977-2016.

3. Francis GS, Siegel RM, Goldsmith SR, Olivari MT, Levine TB, Cohn JN. Acute vasoconstrictor response to intravenous furosemide in patients with chronic congestive heart failure: Activation of the neurohumoral axis. Ann Intern Med 1985; 103: 1 -6.

4. Cooper HA, Dries DL, Davis CE, Shen YL, Domanski MJ. Diuretics and risk of arrhythmic death in patients with left ventricular dysfunction. Circulation 1999; 100: 1311-1315.

5. Lopez B, Querejeta R, Gonzalez A, Sanchez E, Larman M, Diez J. Effects of loop diuretics on myocardial fibrosis and collagen type I turnover in chronic heart failure. J Am Coll Cardiol 2004; 43: 2028 2035.

6. McCurley JM, Hanlon SU, Wei SK, Wedam EF, Michalski M, Haigney MC. Furosemide and the progression of left ventricular dysfunction in experimental heart failure. J Am Coll Cardiol 2004; 44: 1301 1307.

7. Hasselblad V, Gattis Stough W, Shah MR, Lokhnygina Y, O'Connor CM, Califf RM, et al. Relation between dose of loop diuretics and outcomes in a heart failure population: Results of the ESCAPE trial. Eur J Heart Fail 2007; 9: 1064-1069.

8. Ahmed A, Husain A, Love TE, Gambassi G, Dell'Italia LJ, Francis GS, et al. Heart failure, chronic diuretic use, and increase in mortality and hospitalization: An observational study using propensity score methods. Eur Heart J 2006; 27: 1431-1439.

9. Hamaguchi S, Kinugawa S, Goto D, Tsuchihashi-Makaya M, Yokota T, Yamada S, et al. Predictors of long-term adverse outcomes in elderly patients over 80 years hospitalized with heart failure. Circ $J$ 2011; 75: 2403-2410.

10. Tsutsui H, Tsuchihashi-Makaya M, Kinugawa S, Goto D, Takeshita A. Clinical characteristics and outcome of hospitalized patients with heart failure in Japan. Circ J 2006; 70: 1617-1623.

11. Hamaguchi S, Tsuchihashi-Makaya M, Kinugawa S, Yokota T, Ide $\mathrm{T}$, Takeshita A, et al. Chronic kidney disease as an independent risk for long-term adverse outcomes in patients hospitalized with heart failure in Japan: Report from the Japanese Cardiac Registry of Heart Failure in Cardiology (JCARE-CARD). Circ J 2009; 73: 1442-1447.

12. Hamaguchi S, Tsuchihashi-Makaya M, Kinugawa S, Yokota T, Takeshita A, Yokoshiki H, et al. Anemia is an independent predictor of long-term adverse outcomes in patients hospitalized with heart failure in Japan: A report from the Japanese Cardiac Registry of Heart Failure in Cardiology (JCARE-CARD). Circ J 2009; 73: 1901-1908.

13. Hamaguchi S, Yokoshiki H, Kinugawa S, Tsuchihashi-Makaya M, Yokota T, Takeshita A, et al. Effects of atrial fibrillation on longterm outcomes in patients hospitalized for heart failure in Japan: A report from the Japanese Cardiac Registry of Heart Failure in Cardiology (JCARE-CARD). Circ J 2009; 73: 2084-2090.

14. Tsuchihashi-Makaya M, Hamaguchi S, Kinugawa S, Yokota T, Goto $\mathrm{D}$, Yokoshiki H, et al. Characteristics and outcomes of hospitalized patients with heart failure and reduced vs preserved ejection fraction: Report from the Japanese Cardiac Registry of Heart Failure in Car- diology (JCARE-CARD). Circ J 2009; 73: 1893-1900.

15. Hamaguchi S, Kinugawa S, Tsuchihashi-Makaya M, Goto K, Goto D, Yokota T, et al. Spironolactone use at discharge was associated with improved survival in hospitalized patients with systolic heart failure. Am Heart J 2010; 160: 1156-1162.

16. Hamaguchi S, Tsuchihashi-Makaya M, Kinugawa S, Goto D, Yokota $\mathrm{T}$, Goto $\mathrm{K}$, et al. Body mass index is an independent predictor of long-term outcomes in patients hospitalized with heart failure in Japan. Circ J 2010; 74: 2605-2611.

17. Tsuchihashi-Makaya M, Furumoto T, Kinugawa S, Hamaguchi S, Goto K, Goto D, et al. Discharge use of angiotensin receptor blockers provides comparable effects with angiotensin-converting enzyme inhibitors on outcomes in patients hospitalized for heart failure. Hypertens Res 2010; 33: 197-202.

18. Tsuchihashi-Makaya M, Kinugawa S, Yokoshiki H, Hamaguchi S, Yokota T, Goto D, et al. Beta-blocker use at discharge in patients hospitalized for heart failure is associated with improved survival. Circ J 2010; 74: 1364-1371.

19. Hamaguchi S, Furumoto T, Tsuchihashi-Makaya M, Goto K, Goto D, Yokota T, et al. Hyperuricemia predicts adverse outcomes in patients with heart failure. Int J Cardiol 2011; 151: 143-147.

20. Tsuchihashi-Makaya M, Hamaguchi S, Kinugawa S, Goto K, Goto D, Furumoto T, et al. Sex differences with respect to clinical characteristics, treatment, and long-term outcomes in patients with heart failure. Int J Cardiol 2011; 150: 338-339.

21. Rosenbaum PR. Sensitivity to hidden bias. In: Rosenbaum PR, editor. Observational studies. 2nd edn. New York: Springer; 2002; 110124.

22. Eshaghian S, Horwich TB, Fonarow GC. Relation of loop diuretic dose to mortality in advanced heart failure. Am J Cardiol 2006; 97: 1759-1764.

23. Dzau VJ, Colucci WS, Hollenberg NK, Williams GH. Relation of the renin-angiotensin-aldosterone system to clinical state in congestive heart failure. Circulation 1981; 63: 645-651.

24. Packer M. The neurohormonal hypothesis: A theory to explain the mechanism of disease progression in heart failure. J Am Coll Cardiol 1992; 20: $248-254$.

25. Francis GS, Benedict C, Johnstone DE, Kirlin PC, Nicklas J, Liang $\mathrm{CS}$, et al. Comparison of neuroendocrine activation in patients with left ventricular dysfunction with and without congestive heart failure: A substudy of the Studies of Left Ventricular Dysfunction (SOLVD). Circulation 1990; 82: 1724-1729.

26. Weber KT, Brilla CG. Pathological hypertrophy and cardiac interstitium: Fibrosis and renin-angiotensin-aldosterone system. Circulation 1991; 83: 1849-1865.

27. Lijnen P, Petrov V. Induction of cardiac fibrosis by aldosterone. $J$ Mol Cell Cardiol 2000; 32: 865-879.

28. Oudit GY, Kassiri Z, Patel MP, Chappell M, Butany J, Backx PH, et al. Angiotensin II-mediated oxidative stress and inflammation mediate the age-dependent cardiomyopathy in ACE2 null mice. Cardiovasc Res 2007; 75: 29-39.

29. Lopez B, Gonzalez A, Diez J. Circulating biomarkers of collagen metabolism in cardiac diseases. Circulation 2010; 121: 1645-1654.

30. Tokmakova MP, Skali H, Kenchaiah S, Braunwald E, Rouleau JL, Packer M, et al. Chronic kidney disease, cardiovascular risk, and response to angiotensin-converting enzyme inhibition after myocardial infarction: The Survival And Ventricular Enlargement (SAVE) study. Circulation 2004; 110: 3667-3673.

31. Shlipak MG, Smith GL, Rathore SS, Massie BM, Krumholz HM. Renal function, digoxin therapy, and heart failure outcomes: Evidence from the digoxin intervention group trial. J Am Soc Nephrol 2004; 15: 2195-2203.

32. McAlister FA, Ezekowitz J, Tonelli M, Armstrong PW. Renal insufficiency and heart failure: Prognostic and therapeutic implications from a prospective cohort study. Circulation 2004; 109: 1004-1009.

33. Hillege HL, Nitsch D, Pfeffer MA, Swedberg K, McMurray JJ, Yusuf $\mathrm{S}$, et al. Renal function as a predictor of outcome in a broad spectrum of patients with heart failure. Circulation 2006; 113: 671-678.

34. Smith GL, Lichtman JH, Bracken MB, Shlipak MG, Phillips CO, DiCapua P, et al. Renal impairment and outcomes in heart failure: Systematic review and meta-analysis. J Am Coll Cardiol 2006; 47: 1987-1996.

35. Go AS, Yang J, Ackerson LM, Lepper K, Robbins S, Massie BM, et al. Hemoglobin level, chronic kidney disease, and the risks of death and hospitalization in adults with chronic heart failure: The Anemia in Chronic Heart Failure: Outcomes and Resource Utilization (ANCHOR) Study. Circulation 2006; 113: 2713-2723.

36. Heywood JT, Fonarow GC, Costanzo MR, Mathur VS, Wigneswaran JR, Wynne J. High prevalence of renal dysfunction and its impact on outcome in 118,465 patients hospitalized with acute decompensated 
heart failure: A report from the ADHERE database. J Card Fail 2007; 13: $422-430$.

37. Komukai K, Ogawa T, Yagi H, Date T, Sakamoto H, Kanzaki Y, et al. Decreased renal function as an independent predictor of re-hospitalization for congestive heart failure. Circ J 2008; 72: 1152-1157.

38. Shiba N, Matsuki M, Takahashi J, Tada T, Watanabe J, Shimokawa H. Prognostic importance of chronic kidney disease in Japanese patients with chronic heart failure. Circ J 2008; 72: 173-178.

39. Vaz Perez A, Otawa K, Zimmermann AV, Stockburger M, MullerWerdan U, Werdan K, et al. The impact of impaired renal function on mortality in patients with acutely decompensated chronic heart failure. Eur J Heart Fail 2010; 12: 122-128.

40. Robertson JI. Diuretics, potassium depletion and the risk of arrhythmias. Eur Heart J 1984; 5(Suppl A): 25-28.

41. Hollifield JW. Potassium and magnesium abnormalities: Diuretics and arrhythmias in hypertension. Am J Med 1984; 77: $28-32$.
42. Dyckner T. Relation of cardiovascular disease to potassium and magnesium deficiencies. Am J Cardiol 1990; 65: 44K-46K.

43. Dyckner T, Wester PO. Potassium/magnesium depletion in patients with cardiovascular disease. Am J Med 1987; 82: 11-17.

44. Kjeldsen K. Hypokalemia and sudden cardiac death. Exp Clin Cardiol 2010; 15: e96-e99.

45. Dickstein K, Cohen-Solal A, Filippatos G, McMurray JJ, Ponikowski P, Poole-Wilson PA, et al. ESC Guidelines for the diagnosis and treatment of acute and chronic heart failure 2008: The Task Force for the Diagnosis and Treatment of Acute and Chronic Heart Failure 2008 of the European Society of Cardiology. Developed in collaboration with the Heart Failure Association of the ESC (HFA) and endorsed by the European Society of Intensive Care Medicine (ESICM). Eur Heart J 2008; 29: 2388-2442.

46. America HFSO. Disease management in heart failure. $J$ Card Fail 2006; 12: e58-e69. 